\title{
Renal-Protective Effects and Potential Mechanisms of Traditional Chinese Medicine after Ischemia-Reperfusion Injury
}

\author{
Demin Liu, ${ }^{1}$ Songling Tang, ${ }^{2}$ Lu Gan, ${ }^{2}$ and Wei Cui ${ }^{1}$ \\ ${ }^{1}$ Cardiology Department, Second Hospital of Hebei Medical University, Shijiazhuang, Hebei 050000, China \\ ${ }^{2}$ Research Laboratory of Emergency Medicine, Department of Emergency Medicine, \\ National Clinical Research Center for Geriatrics, West China Hospital, Sichuan University, Chengdu 610041, China \\ Correspondence should be addressed to Wei Cui; cuiweihb2h@163.com
}

Received 9 January 2021; Revised 21 January 2021; Accepted 30 January 2021; Published 19 February 2021

Academic Editor: Zhiqian Zhang

Copyright (C) 2021 Demin Liu et al. This is an open access article distributed under the Creative Commons Attribution License, which permits unrestricted use, distribution, and reproduction in any medium, provided the original work is properly cited.

Renal ischemia-reperfusion (I/R) injury mainly causes acute kidney injury (AKI) after renal transplantation, trauma, sepsis, and hypovolemic shock. Patients with renal I/R injury are frequently associated with a poor prognosis. Traditional Chinese medicine (TCM) has been used for the prevention and treatment of various diseases in China and other Asian countries for centuries. Many studies have shown the protective effect of TCM on renal I/R injury, due to its diverse bioactive components. The potential mechanisms of TCMs on renal I/R injury include anti-inflammation, antioxidative effect, anti-cell death, downregulation of adhesion molecule expression, regulation of energy metabolism by restoring $\mathrm{Na}^{+}-\mathrm{K}^{+}$-ATPase activity, and mitochondrial fission. This review summarizes the major developments in the effects and underlying mechanisms of TCMs on the renal I/R injury.

\section{Introduction}

Renal ischemia-reperfusion (I/R) injury is the main cause of acute kidney injury (AKI) and is reported to be associated with high morbidity and mortality in both adults and children. It often occurs after renal transplantation, as well as in sepsis, trauma, hypovolemic shock, etc. [1]. During the renal I/R process, excess reactive oxygen species (ROS) are likely to cause oxidative stress, which subsequently triggers lipid peroxidation [2] and cell death due to apoptosis, autophagy, pyroptosis, and ferroptosis caused by DNA and protein damage in ischemic tissue [3]. In addition, inflammation plays an important role in renal I/R injury, along with leukocyte infiltration and tissue damage in the kidney, followed by excessive production of proinflammatory cytokines [4]. Energy metabolism dysfunction, such as decreased $\mathrm{Na}^{+}-\mathrm{K}^{+}$-ATPase activity, has been considered as one of the critical mechanisms of renal I/R injury [5].

Increase in blood urea nitrogen (BUN) and creatinine (Cr) [3] and a higher urinary total protein level are reported to occur after renal I/R injury [6]. Histopathology of renal I/ $\mathrm{R}$ injury shows luminal narrowing, intraluminal necrotic cellular debris, tubular basal membrane rupture, tubular vacuolization, interstitial congestion, apparent cell swelling and nuclear infiltration in the H\&E staining of renal tissues [7], and higher Kim-1 expression, thus causing a higher renal tubular injury score [2].

Traditional Chinese medicine (TCM) has been used in China, Korea, Japan, and other Asian countries for the treatment of various diseases, such as cerebrovascular diseases, cardiac diseases, liver diseases, renal disorders, etc. In the theory of TCM, renal I/R injury is attributed to Qi and blood deficiency and blood stasis syndrome [8]. From the perspective of TCM, renal I/R injury should be treated through the replenishment of Qi and activation of blood circulation [7]. Several TCMs have been applied for the prevention and treatment of renal $\mathrm{I} / \mathrm{R}$, and they show different renal-protective effects in Table 1, including antiinflammation (Cordyceps sinensis, cordycepin, Yisheng injection, cryptotanshinone, Panax notoginseng, total glucosides of paeony, hydroxysafflor yellow A, farnesiferol B, anemoside B4, etc.), antioxidative stress (ligustrazine, berberine, glycyrrhizin, Sheng Mai San, hyperoside, salvianolic acid A, salvianolic acid B, arctigenin, farnesiferol B, 
TABLE 1: The protective effects and potential mechanisms of TCM and the major ingredients on renal I/R injury.

\begin{tabular}{|c|c|c|c|c|}
\hline TCM or its ingredients & Experiment object & Protective effects & Potential mechanisms & Ref. \\
\hline Cordyceps sinensis & SD rats & Decreased inflammation & - & [9] \\
\hline Cordycepin & SD rats & $\begin{array}{c}\text { Decreased inflammation, apoptosis, oxidative } \\
\text { stress }\end{array}$ & Nrf2-HO-1 & {$[10]$} \\
\hline Ligustrazine & C57BL/6 mice & Decreased apoptosis, oxidative stress & - & [11] \\
\hline Yisheng injection & C57BL/6 mice & $\begin{array}{c}\text { Reduced neutrophil infiltration, decreased } \\
\text { inflammation }\end{array}$ & - & [12] \\
\hline \multirow{3}{*}{$\begin{array}{l}\text { Fufang Shenhua } \\
\text { Tablet }\end{array}$} & Wistar rats & Decreased inflammation & Downregulation of TLRs & {$[8]$} \\
\hline & Wistar rats & Decreased inflammation & MyD88 signaling pathway & [13] \\
\hline & Wistar rats & Decreased $\mathrm{Na}+-\mathrm{K}+-\mathrm{ATPase}$ level & - & {$[5]$} \\
\hline Berberine & NRK-52E cells & $\begin{array}{l}\text { Decreased mitochondrial oxidative stress and } \\
\text { apoptosis }\end{array}$ & Sirt1/p53 signaling pathway & [14] \\
\hline Glycyrrhizin & C57BL/6 mice & $\begin{array}{l}\text { Inhibition of inflammation and renal cell } \\
\text { apoptosis }\end{array}$ & $\begin{array}{l}\text { p38 MAPK signaling } \\
\text { pathway }\end{array}$ & {$[15]$} \\
\hline Honokiol & Wistar albino rats & Inhibition of oxidative stress and inflammation & - & [16] \\
\hline Cryptotanshinone & C57BL/6 mice & $\begin{array}{c}\text { Inhibition of cell apoptosis and inflammatory } \\
\text { response }\end{array}$ & $\begin{array}{c}\text { NF- } \kappa \text { B-p38 MAPK signaling } \\
\text { pathway }\end{array}$ & [17] \\
\hline Hyperoside & $\begin{array}{l}\text { C57BL/ } 6 \text { mice } \\
\text { HK-2 cells }\end{array}$ & $\begin{array}{c}\text { Attenuated tubular cell apoptosis, oxidative stress, } \\
\text { inhibited mitochondrial fission }\end{array}$ & OMA1-OPA1 axis & [18] \\
\hline Notoginsenoside R1 & SD rats & Reduced apoptosis and inflammatory response & $\begin{array}{c}\text { NF- } \kappa \text { B-p38 MAPK signaling } \\
\text { pathway }\end{array}$ & [19] \\
\hline Brazilin & SD rats & & NF- $\kappa$ B signaling pathway & {$[20]$} \\
\hline Salvianolic acid A & $\begin{array}{l}\text { SD rats } \\
\text { HK-2 cells }\end{array}$ & and oxidative stress & Akt/mTOR/4EBP1 pathway & [21] \\
\hline Salvianolic acid B & $\mathrm{BALB} / \mathrm{c}$ mice & $\begin{array}{l}\text { Reduced oxidative stress and inflammation, } \\
\text { inhibition of pyroptosis }\end{array}$ & $\begin{array}{l}\text { Nrf2/NLRP3 signaling } \\
\text { pathway }\end{array}$ & [22] \\
\hline $\begin{array}{l}\text { Total glucosides of } \\
\text { paeony }\end{array}$ & $\begin{array}{c}\text { SD rats } \\
\text { NRK-52E cells }\end{array}$ & Attenuated apoptosis and inflammation & $\begin{array}{l}\mathrm{XIST} / \mathrm{miR}-124-3 \mathrm{p} / \mathrm{ITGB} 1 \\
\text { axis }\end{array}$ & [23] \\
\hline $\begin{array}{l}\text { Hydroxysafflor yellow } \\
\text { A }\end{array}$ & $\mathrm{SD}$ rats & Decreased inflammation & TLR4/NF- $\kappa$ B pathway & {$[4]$} \\
\hline Sanqi oral solution & $\mathrm{SD}$ rats & Enhanced autophagy, attenuated apoptosis & ERK/mTOR pathway & [7] \\
\hline Arctigenin & C57BL/6 mice & $\begin{array}{l}\text { Alleviated inflammatory response and oxidative } \\
\text { stress }\end{array}$ & NF- $\kappa \mathrm{B}$ signaling pathway & [3] \\
\hline Farnesiferol B & Female C57/BJ mice & Attenuated inflammation & $\begin{array}{l}\text { TGR5/NF- } \kappa \mathrm{B} \text { signaling } \\
\text { pathway }\end{array}$ & {$[2]$} \\
\hline Polydatin & $\begin{array}{c}\text { BALB/c mice } \\
\text { Primary renal tubular } \\
\text { epithelial cells }\end{array}$ & Antiapoptosis and antioxidative effects & $\begin{array}{l}\text { Sonic hedgehog (shh) } \\
\text { signaling pathway }\end{array}$ & [24] \\
\hline
\end{tabular}

polydatin, Shenfu injection, etc.), anti-cell death (honokiol, cryptotanshinone, brazilin, salvianolic acid B, Sanqi oral solution, etc.), downregulation of adhesion molecule expression (ligustrazine and Yisheng injection), regulation of energy metabolism by restoring $\mathrm{Na}^{+}-\mathrm{K}^{+}$-ATPase activity (Fufang Shenhua Tablet), and mitochondrial fission (hyperoside).

In this review, we have discussed the studies in the past decades on the protective effects and potential mechanisms of TCMs and the major ingredients on renal I/R injury.

\section{Protective Effects of TCMs and Major Ingredients on Renal I/R Injury}

Generally speaking, almost all the following TCMs and the major ingredients play a protective role in renal I/R injury, including the improvement of renal function and histological changes in renal tissues, inhibiting inflammatory cytokine release and macrophage/neutrophil infiltration, decreasing production of oxidative stress and lipid oxidation, regulating programmed cell death (apoptosis, autophagy, pyroptosis, or ferroptosis), decreasing release of adhesion molecules, regulating energy metabolism, endothelial injury, and mitochondrial dysfunction [25].

\subsection{Renal Function and Renal Histological Examination.} In previous studies, renal function was often measured by the levels of Scr and BUN. In some cases, urinary total protein level was also used for renal function measurement. It was reported that $\mathrm{Scr}$ and BUN levels were significantly increased in the I/R model group, compared with the sham group. TCMs or CCMs, such as Fufang Shenhua Tablet, cryptotanshinone, and notoginsenoside R1, could inhibit the increased levels of Scr and BUN, and thus improve renal function $[13,17,19]$. In addition, anemoside B4 could decrease the urinary total protein level [6], indicating improvement of renal function.

Several histological abnormalities were observed after renal I/R injury, including tubular brush border loss, epithelial cell dilatation and necrosis, cytoplasmic 
vacuolization, and cast formation [3]. Most TCMs were reported to alleviate renal histological changes and decrease renal tubular injury score, caused by I/R injury. Furthermore, TCMs, such as Sanqi oral solution, hydroxysafflor yellow A, and total glucosides of paeony, showed dosedependent effects, such that higher concentrations showed greater alleviating effects $[4,7,23]$. In contrast, ATG played a detrimental role in renal $\mathrm{I} / \mathrm{R}$ injury since renal function and histology worsened with increasing concentration [3].

2.2. Anti-Inflammatory Activity. Most TCMs play an antiinflammatory role in renal protection after I/R injury. Increase in expressions of inflammatory genes, such as MCP-1 and TNF-a mRNA, and their proteins were observed in the renal I/R group. Cordyceps sinensis treatment was reported to reverse these effects $[9,26]$. Additionally, cordycepin, the extract from Cordyceps sinensis, could decrease secretion of proinflammatory factors and alleviate inflammatory reaction [10], including IL- $\beta$, IL- 6 , and TNF- $\alpha$, at the protein and mRNA levels. The same effect was observed after treatment with Yisheng injection, Fufang Shenhua Tablet, glycyrrhizin, honokiol, cryptotanshinone, notoginsenoside R1, brazilin, total glucosides of paeony, hydroxysafflor yellow $\mathrm{A}$, arctigenin, farnesiferol $\mathrm{B}$, and anemoside $\mathrm{B} 4$ $[2-4,6,8,12,13,15-17,19,20,23]$. Moreover, the infiltration of neutrophils and macrophages was observed in some studies. ATG decreased the infiltration of $\mathrm{CD} 11 \mathrm{~b}+\mathrm{Gr} 1+$ neutrophils and CD68+ macrophages in renal tissue after I/R injury [3], and the neutrophil infiltration was decreased after pretreatment with Yisheng injection [12].

2.3. Antioxidative Stress. Oxidative stress and lipid oxidation increase after renal I/R injury, while treatment with some TCMs inhibits the production of oxidative stress and promotes the production of antioxidant factors. In Feng Han and AR Shahed's studies [10,26], Cordyceps sinensis and its extract cordycepin increased the levels of antioxidative factors, including GSH, GSHPx, and SOD, and decreased the levels of oxidative stress products, including MDA, NO, and iNOS. Other TCMs also showed similar effects, such as MDA decrease and SOD increase in the ligustrazine treatment group [11], berberine treatment group, and Sal A and Sal B treatment groups [21, 22], SOA decrease and SOD increase after glycyrrhizin treatment [15], MDA/MPO decrease and SOD/CAT increase after honokiol treatment [16], decrease in iNOS and $\mathrm{ONOO}^{-}$levels after SMS treatment [27], and decrease in ROS levels after hyperoside treatment. CS treatment decreased NGAL levels both in kidney tissues and in urine, which was increased in the I/R group [28]. In addition, farnesiferol $B$ reduced oxidative stress (diminished levels of NAGL and $\mathrm{H} 2 \mathrm{O} 2$ ) and lipid oxidative signaling pathways (diminished levels of lipid peroxidation markers, including 4-HNE and MDA) in renal I/R [2].

2.4. Anti-Cell Death. There are several types of programmed cell death, including apoptosis, autophagy, pyroptosis, and ferroptosis. Previous studies have reported that the protective effect of TCMs on renal I/R injury was associated with programmed cell death, especially apoptosis.

Antiapoptosis was observed after treatment with some TCMs before or after renal I/R injury, with downregulation of the levels of apoptosis proteins and upregulation of the levels of antiapoptosis proteins. In the Cordyceps sinensis treatment group [26], Fas and FasL mRNA expression levels were decreased, while caspase- 3 activity was decreased after glycyrrhizin and honokiol treatment $[15,16]$. TUNELpositive cells were increased after Sal A treatment, indicating a reduction of apoptotic activity [21]. An in vitro study found that berberine significantly inhibited the expression of apoptotic proteins, such as Bax [14].

Treatment with Sanqi (SQ) oral solution showed decrease in apoptosis and increase in autophagy [7]. Lower Bax and caspase-3 levels and higher Bcl-2 level were observed in the SQ treatment group in a dose-dependent manner, demonstrating the antiapoptotic activity of SQ. Meanwhile, compared to the I/R model group, higher dose of SQ significantly increased the levels of LC3II/LC3I and Beclin1, indicating an enhanced autophagy activity after renal I/R injury. In addition, treatment with SQ and 3-MA, an autophagy inhibitor, showed worse histological damages and renal function, compared with SQ alone treatment, indicating the essential role of autophagy in SQ treatment during renal $\mathrm{I} / \mathrm{R}$ injury.

In Yu Pang's study [22], the levels of GSDMD, caspase-1, and IL-1 $\beta$, the pyroptosis-related proteins, were strongly upregulated in the model group. The pyroptosis-related proteins were significantly decreased after treatment with Sal $\mathrm{B}$, demonstrating the antipyroptosis activity of Sal B.

Lastly, ferroptosis plays an important role in the protective effects of farnesiferol B treatment on renal I/R injury. The mRNA expression of Gpx4, the key ferroptosis regulator, was significantly reduced after renal I/R injury, while farnesiferol B treatment increased its expression. However, the intergroup difference was not significant [2]. So, further studies are needed.

2.5. Downregulation of Adhesion Molecules. In the I/R model group, expression of ICAM-1 was upregulated but was greatly diminished after ligustrazine treatment [11]. Moreover, ICAM-1 was downregulated in a dose-dependent manner after pretreatment with Yisheng injection in the renal I/R group [12].

2.6. Recovery of $\mathrm{Na}^{+}-\mathrm{K}^{+}$-ATPase Activity. Yang Yang et al. [5] found a significant decrease in $\mathrm{Na}^{+}-\mathrm{K}^{+}$-ATPase activity in the I/R model group when compared with the sham group. However, Shenhua Tablet (SHT) was observed to increase the decreased $\mathrm{Na}^{+}-\mathrm{K}^{+}$-ATPase activity after renal I/ $\mathrm{R}$, both in the low-dose group and the high-dose group. Combined with other results in this study, the authors concluded that SHT could recover the number of $\mathrm{Na}^{+}-\mathrm{K}^{+}$ATPase and improve its activity in the tubular epithelial cells, promote epithelial cell polarity in renal tubular cells, and thereby improve renal function. 
2.7. Endothelial Protective Effects. Salvianolic acid A (SAA) can protect peritubular capillary endothelium from renal I/R injury [29]. VEGFA expression was increased in tubular epithelial cells, which was associated with peritubular capillary density in the SAA treatment group, as well as the reduced levels of plasma $\mathrm{vWF}$ and lower platelet activation after I/R injury. Moreover, significant increase in Klotho protein expression was observed, indicating less endothelial injury after renal I/R with SAA treatment.

\section{Potential Mechanism of TCMs and Major Ingredients in Renal I/R Injury}

Several signaling pathways were considered to be involved in the renoprotective effects of TCMs, especially the anti-inflammation effect, antioxidative effect, antiapoptotic activity, and some others. These pathways included the NF- $\kappa \mathrm{B} / \mathrm{TLR} 4$, NF- $\kappa$ B-p65, NF- $\kappa$ B-p MAPK, Nrf2-NLRP3, Nrf2-HO-1, Shh signaling pathway, PI3K-Akt [30], ERK/mTOR [7], Akt/ mTOR/4EBP1 [21], OMA1-OPA1 [18], XIST/MicroRNA124-3p/ITGB1 [23], etc. The most important pathways involved in renal I/R injury are discussed below.

3.1. NF- $\kappa B$ Signaling Pathway. NF- $\kappa \mathrm{B}$ is believed to play an important role in the anti-inflammatory effects and antiapoptotic and antioxidative activity of TCMs in renal I/R process.

Activation of the NF- $\kappa \mathrm{B}$ signaling pathway was inhibited in the cryptotanshinone (CTS) treatment group after renal I/ $\mathrm{R}$ [17]. I/R significantly increased the phosphorylated p65 and $\mathrm{I} \kappa \mathrm{B} \alpha$ levels, indicating the activation of NF- $\kappa \mathrm{B}$ signaling pathway. After CTS treatment, the phosphorylation of p65 and $\mathrm{I} \kappa \mathrm{B} \alpha$ was inhibited. Brazilin was also proven to downregulate the inflammatory activity by inhibiting NF- $\kappa \mathrm{B}$ signaling pathway activation in vitro [20].

Hydroxysafflor yellow A (HSYA) was reported to decrease the renal inflammation after I/R injury by suppressing the TLR4/NF- $\kappa \mathrm{B}$ signaling pathway activation [4], as phospho-IKK $\beta$, phospho-I $\kappa \mathrm{B} \alpha$, and NF- $\kappa \mathrm{B}-\mathrm{p} 65$ expression levels were upregulated in the I/R group but were downregulated after treatment with HSYA in vitro. Similar effect of arctigenin (ATG) and polydatin on TLR4 and NF- $\kappa$ B-p65 expression was observed [3, 31].

Notoginsenoside R1 (NR1) decreased inflammatory factors and apoptosis by inhibiting the phosphorylation of p38 MAPK and NF- $\kappa$ B activation in renal tissue after I/R injury [12]. The anti-inflammatory effects and antioxidative activities of farnesiferol $B$ on renal I/R injury were based on the activation of TGR 5 and inhibition of the NF- $\kappa$ B pathway [2].

3.2. Nrf2 Signaling Pathway. In renal I/R process, Nrf2 plays a central role in renal-protective effects of TCMs, including the anti-inflammatory effects and antioxidative and anti-cell death activity.

Cordycepin is known to alleviate inflammation, oxidative stress, and apoptosis in the I/R process. The antioxidative mechanism of cordycepin was analyzed by measuring the levels of Nrf2 and HO-1, the key proteins in antioxidative stress process. Western blotting results showed an increase in the expression of $\mathrm{Nrf} 2$ and $\mathrm{HO}-1$ after cordycepin treatment, indicating that cordycepin may suppress the oxidative stress during renal I/R injury through the Nrf2/ HO-1 signaling pathway [10].

Another study found that Sal B could inhibit caspase-1/ GSDMD-mediated pyroptosis and thereby alleviate I/R injury in mice, by activating Nrf2/NLRP3 signaling pathway [22]. Sal B was reported to inhibit pyroptosis by downregulating pyroptosis-related proteins GSDMD, caspase-1, and IL- $1 \beta$. Moreover, Sal B showed its antioxidative property by inhibiting the expression of Keap1 protein, thus increasing Nrf2 nuclear expression and HO-1 protein levels in vivo. In vitro, Sal B reduced NLRP3 protein expression and increased Nrf2 nuclear import and activated expression of downstream antioxidant components. Moreover, the Nrf2/ NLRP3 signaling pathway was demonstrated to be involved through Nrf2 knockdown in vivo and siNrf2 transfection in vitro.

3.3. Sonic Hedgehog (Shh) Signaling Pathway. Shh signaling pathway is important in the antioxidative and antiapoptotic effects of TCM treatment after renal I/R injury. Polydatin alleviated apoptosis and oxidative stress after renal I/R injury through activating the sonic hedgehog signaling pathway [24]. Polydatin was found to dose-dependently induce increase in the Shh gene and protein expression compared with the I/R group, both in vitro and in vivo. Moreover, the mRNA and protein expression of Ptch 1 and Smo, two key elements in the Shh signaling pathway, were reduced by cyclopamine (an inhibitor of Smo) combined with polydatin. Meanwhile, higher apoptotic activity and oxidative effects were observed. Therefore, inhibition of Shh signaling impaired the antioxidative and antiapoptotic effects of polydatin.

\section{Summary}

This review discusses the protective effects of TCMs and their active components on renal I/R injury. The mechanisms of the protective effects of TCMs include inhibition of inflammation, decrease of oxidative stress and lipid oxidation products, regulation of programmed cell death, reduction of adhesion molecule release, and regulation of energy metabolism and endothelial injury by activating NF$\kappa \mathrm{B}$ and Nrf2 signaling pathways, which are considered to be multiple targets for successful treatment. However, the existing studies are either animal experiments in vivo or in vitro studies, with lower level of evidence and strength of recommendation. Therefore, further clinical studies are needed to explore the effects of TCMs and the underlying mechanisms.

\section{Conflicts of Interest}

The authors declare that they have no conflicts of interest. 


\section{Authors' Contributions}

Demin Liu and Songling Tang contributed equally to this study.

\section{References}

[1] Z. Sun and X. Wang, "Protective effects of polydatin on multiple organ ischemia-reperfusion injury," Bioorganic Chemistry, vol. 94, Article ID 103485, 2020.

[2] L. Zhang, X. Fu, T. Gui et al., "Effects of Farnesiferol B on ischemia-reperfusion-induced renal damage, inflammation, and NF- $\kappa \mathrm{B}$ signaling," International Journal of Molecular Sciences, vol. 20, 2019.

[3] F. Han, X.-X. Xia, M. Dou et al., "Arctigenin: a two-edged sword in ischemia/reperfusion induced acute kidney injury," Biomedicine \& Pharmacotherapy, vol. 103, pp. 1127-1136, 2018.

[4] J. Bai, J. Zhao, D. Cui et al., "Protective effect of hydroxysafflor yellow A against acute kidney injury via the TLR4/NF- $\kappa \mathrm{B}$ signaling pathway," Scientific Reports, vol. 8, p. 9173, 2018.

[5] Y. Yang, R.-B. Wei, X.-Y. Zheng et al., "Effects of compound Shenhua tablet on renal tubular $\mathrm{Na}+\mathrm{K}+-\mathrm{ATPase}$ in rats with acute ischemic reperfusion injury," Chinese Journal of Integrative Medicine, vol. 20, no. 3, pp. 200-208, 2014.

[6] J. Li, S. S. Zuo, X. X. Qiu et al., "[Study on therapeutic effect and its related mechanism of anemoside B4 on ischemia reperfusion injury induced by renal artery and vein ligation in rats]," Zhongguo Zhong Yao Za Zhi, vol. 45, pp. 617-622, 2020.

[7] R. Tian, P. Wang, L. Huang et al., "Sanqi oral solution ameliorates renal ischemia/reperfusion injury via reducing apoptosis and enhancing autophagy: involvement of ERK/ mTOR pathways," Frontiers in Pharmacology, vol. 11, Article ID 537147, 2020.

[8] X.-Y. Zheng, R.-B. Wei, S.-Z. Shi, Z. Yin, and X.-M. Chen, "Effects of Fufang Shenhua tablet on the expression of toll-like receptors during acute kidney injury induced by ischemiareperfusion in rats," Chinese Journal of Integrative Medicine, vol. 18, no. 12, pp. 918-924, 2012.

[9] H.-P. Wang, C.-W. Liu, H.-W. Chang, J.-W. Tsai, Y.-Z. Sung, and L.-C. Chang, "Cordyceps sinensis protects against renal ischemia/reperfusion injury in rats," Molecular Biology Reports, vol. 40, no. 3, pp. 2347-2355, 2013.

[10] F. Han, M. Dou, Y. Wang et al., "Cordycepin protects renal ischemia/reperfusion injury through regulating inflammation, apoptosis, and oxidative stress," Acta Biochimica et Biophysica Sinica, vol. 52, no. 2, pp. 125-132, 2020.

[11] L. Feng, Y. Xiong, F. Cheng, L. Zhang, S. Li, and Y. Li, "Effect of ligustrazine on ischemia-reperfusion injury in murine kidney," Transplantation Proceedings, vol. 36, no. 7, pp. 1949-1951, 2004.

[12] L. Feng, Y. Guo, F. Cheng et al., "Effects of pretreatment with Yisheng injection on renal warm ischemia/reperfusion injury in mice," Transplantation Proceedings, vol. 42, no. 5, pp. 1545-1549, 2010.

[13] Q.-P. Li, R.-B. Wei, X. Yang et al., "Protective effects and mechanisms of Shenhua tablet (肾华片) on toll-like receptors in rat model of renal ischemia-reperfusion injury," Chinese Journal of Integrative Medicine, vol. 25, no. 1, pp. 37-44, 2019.

[14] Y. Lin, M. Sheng, Y. Ding et al., "Berberine protects renal tubular cells against hypoxia/reoxygenation injury via the Sirt1/p53 pathway," Journal of Natural Medicines, vol. 72, no. 3, pp. 715-723, 2018.
[15] S. Ye, Y. Zhu, Y. Ming, X. She, H. Liu, and Q. Ye, "Glycyrrhizin protects mice against renal ischemia-reperfusion injury through inhibition of apoptosis and inflammation by downregulating p38 mitogen-activated protein kinase signaling," Experimental and Therapeutic Medicine, vol. 7, no. 5, pp. 1247-1252, 2014.

[16] Y. Yu, M. Li, N. Su et al., "Honokiol protects against renal ischemia/reperfusion injury via the suppression of oxidative stress, iNOS, inflammation and STAT3 in rats," Molecular Medicine Reports, vol. 13, no. 2, pp. 1353-1360, 2016.

[17] T. Bai, K. Yang, C. Qin, T. Xu, X. Yu, and J. Zhang, "Cryptotanshinone ameliorates renal ischaemia-reperfusion injury by inhibiting apoptosis and inflammatory response," Basic \& Clinical Pharmacology \& Toxicology, vol. 125, no. 5, pp. 420-429, 2019.

[18] L. Wu, Q. Li, S. Liu et al., "Protective effect of hyperoside against renal ischemia-reperfusion injury via modulating mitochondrial fission, oxidative stress, and apoptosis," Free Radical Research, vol. 53, no. 7, pp. 727-736, 2019.

[19] W.-J. Liu, H.-T. Tang, Y.-T. Jia et al., "Notoginsenoside R1 attenuates renal ischemia-reperfusion injury in rats," Shock, vol. 34, no. 3, pp. 314-320, 2010.

[20] Y. Jia, J. Zhao, M. Liu et al., "Brazilin exerts protective effects against renal ischemia-reperfusion injury by inhibiting the NF- $\kappa$ B signaling pathway," International Journal of Molecular Medicine, vol. 38, no. 1, pp. 210-216, 2016.

[21] Y. Song, W. Liu, Y. Ding et al., "Salvianolic acid A ameliorates renal ischemia/reperfusion injury by activating Akt/mTOR/ 4EBP1 signaling pathway," American Journal of PhysiologyRenal Physiology, vol. 315, no. 2, pp. F254-f262, 2018.

[22] Y. Pang, P. C. Zhang, R. R. Lu et al., "Andrade-oliveira salvianolic acid $B$ modulates caspase-1-mediated pyroptosis in renal ischemia-reperfusion injury via Nrf2 pathway," Frontiers in Pharmacology, vol. 11, Article ID 541426, 2020.

[23] F. Chen, Y. Hu, Y. Xie et al., "Total glucosides of paeony alleviate cell apoptosis and inflammation by targeting the long noncoding RNA XIST/MicroRNA-124-3p/ITGB1 axis in renal ischemia/reperfusion injury," Mediators of Inflammation, vol. 2020, Article ID 8869511, 13 pages, 2020.

[24] Q.-H. Meng, H.-B. Liu, and J.-B. Wang, "Polydatin ameliorates renal ischemia/reperfusion injury by decreasing apoptosis and oxidative stress through activating sonic hedgehog signaling pathway," Food and Chemical Toxicology, vol. 96, pp. 215-225, 2016.

[25] Z. Zeng, Z. Chen, S. Xu et al., "Polydatin protecting kidneys against hemorrhagic shock-induced mitochondrial dysfunction via SIRT1 activation and p53 deacetylation," Oxidative Medicine and Cellular Longevity, vol. 2016, Article ID 1737185, 15 pages, 2016.

[26] A. R. Shahed, S. I. Kim, and D. A. Shoskes, "Down-regulation of apoptotic and inflammatory genes by Cordyceps sinensis extract in rat kidney following ischemia/reperfusion," Transplantation Proceedings, vol. 33, no. 6, pp. 2986-2987, 2001.

[27] I. Lee, C. Lee, C. Chang, C. Chien, and M. Lin, "Sheng mai san, a Chinese herbal medicine, protects against renal ischaemic injury during heat stroke in the rat," Clinical and Experimental Pharmacology and Physiology, vol. 32, no. 9, pp. 742-748, 2005.

[28] H. Yu, Q. Zhou, R. Huang, M. Yuan, X. Ao, and J. Yang, "[Effect of cordyceps sinensis on the expression of HIF- $1 \alpha$ and NGAL in rats with renal ischemia-reperfusion injury]," Zhong Nan Da Xue Xue Bao Yi Xue Ban, vol. 37, pp. 57-66, 2012. 
[29] Z. Zhang, D. Qi, X. Wang et al., "Protective effect of Salvianolic acid A on ischaemia-reperfusion acute kidney injury in rats through protecting against peritubular capillary endothelium damages," Phytotherapy Research, vol. 32, no. 1, pp. 103-114, 2018.

[30] H. B. Liu, Q. H. Meng, C. Huang, J. B. Wang, and X. W. Liu, "Nephroprotective effects of polydatin against ischemia/ reperfusion injury: a role for the PI3K/Akt signal pathway," Oxidative Medicine and Cellular Longevity, vol. 2015, Article ID 362158, 13 pages, 2015.

[31] Y. Li, W. J. Xiong, J. Yang et al., "[Inhibitory effect of polydatin on expression of toll-like receptor 4 in ischemiareperfusion injured NRK-52E cells]," Zhongguo Zhong Yao Za Zhi, vol. 39, pp. 3157-3161, 2014. 